\title{
ON SOME STATISTICAL PROBLEMS CONNECTED WITH EFFICIENT DESIGN OF TESTS ${ }^{\star}$
}

\author{
Bishwa Nath MUKHERJEE \\ University of North Carolina, Chapel Hill
}

\section{INTRODU'CTION}

In the construction and efficient design of psychological tests, the psychometrician often faced with a number of problems. Among these, problems like (a) determination of optimal test length for maximum differential prediction [Horst, (30)] and difficulties in differential prediction [Brogden, $(8,9)$ ], (b) estimation of test reliability under various conditions and their meaning [Thorndike, (55)] (c) evaluation of various item analysis procedures [Guilford, (25)] and item-selection procedures for maximizing test homogeneity and validity [Webster, $(61,62)$ ], (d) the effect of various principles of item construction on item and test parameters [16], (e) methods of constructing unidimensional tests and their relative merits [42], (f) use of factor analysis [26] and component of variance analysis [8] in test development program, (g) methods of differential weighting of items [34] or criterion keying [22] and the like, now appear clearly formulated and to a great extent solved. Gulliksen [27] has discussed some of these problems and as such no attempt will be made to reiterate them. Problems arising from errors of measurement will not be also discussed since a recent article by Lord [41] covers the area. The purpose of the paper is to focus attention on some of the unsolved statistical problems and a few difficulties that are being encountered currently in the field of test construction. These problems though overlapping in many ways, are discussed under three arbitrary heads, namely, (a) problems in item analysis, (b) problems in determining the various measures of worth for evaluating a test, and lastly (c) problems viewed in the modern decision theory framework.

\section{Problems in Item Analysis}

Guilford [25, p.435] has already discussed some problems of special nature in the field of item analysis such as the effect of speed upon item indices, effect of chance success on item analysis, the problem of shrinkage in the total score reliability of the test due to spurious item-total correlations. Without repeating those, an attempt will be made here to discuss only three problems in item analysis. These problems are connected with (i) adequate way of summarizing item information, (ii) the estimation of item parameters and (iii) item selection procedure under different probability structures relating a set of test responses to the unobservable ability or trait.

Adequate way of summarizing item information:

Two item characteristics are usually very popular as parameters in the joint distri-

The author wishes to express his appreciation to Dr. R. Darrell Bock for his critical reading of the manuscript. 
bution of the items. They are (i) difficulty value of each of the items which can be written as $P_{i}$ and (ii) correlation between pairs of item responses, $\varrho_{i j}$. Correlation of the item scores with the total scores, $\varrho_{i x}$, is also taken into account in order to attain internal consistency. Whenever it is possible to obtain a good external criterion which as a high degree of relevance with the trait under measurement, care is taken to obtain a measure of relationship between the external criterion and the individual items. This parameter is known as item validity. Recently Gadel [23] has suggested another item characteristic, namely curvilinearity of response distributions which applies only to questionnaire items having at least three possible responses. When this characteristic is considered along with item validity, the chance of selecting 'truly' valid items increases. Another way of representing item characteristics is by way of plotting $P_{i}$ as a function of the underlying trait, $Y$ where the latent variable $Y$ is assumed to be monotonically related to the observed total score $x$ so that examinees having identical scores in test $X$ may be treated for practical purposes as having identical value of $Y$. This curve known as item characteristic curve (ICC) or trace line of the item shows the probability of the item being responded positively at different levels on the latent trait continuum. The ICC has the advantage of stating the difficulty value as well as the discriminating power on the same scale since the slope at a point of the curve indicates the precision with which the item discriminates between individuals at that point.

Traditionally some indices of item difficulty as well as inter-item correlation and either item-total correlation or item-criterion correlation in the form of product moment, point biserial, biserial, tetrachoric or phi-coefficients are used to denote item characteristics. From the reports of Gibbons [24] and Carter [12], it seems clear that indices of item difficulty are much more consistent or stable than the indices of item discrimination. Even with respect to item difficulty index, Lord [41] has observed that the proportion of test questions answered correctly by a group of examinees is by itself not a consistent estimate of the corresponding population proportion. However, as Guilford [25] has said, the stability of item indices as well as their precision will depend upon the type of test used and the population tested. It would, therefore be necessary to study further the properties of different types of item indices for various kinds of test items administered to different populations.

\section{Estimation of Item Parameters:}

The estimation of the difficulty level of free response items is a very easy task but this job becomes complicated for multiple choice items where the guessing factor operates in different degrees. Previous workers attacked this problem by making some form of correction for guessing. Guilford [25, p.420] has presented a formula by means of which the corrected proportion can be computed directly from respose count data. In this formula, the assumption essentially is that the choices are equally attractive to the examiness who do not know the correct answer and make a guess. This assumption is not very realistic. A more realistic model was proposed by Horst [29] nearly thirty years back. Horst assumed that those examinees not knowing the correct answer can be grouped under various categories. The first category is composed of those who know nothing about the adequacy of the alternatives in which case the choices will be equally distributed to 
all the $f$ possible answers. The second category is composed of those examinees who know that one of the alternatives is wrong and as such they will distribute uniformly their answers over the remaining $(f-1)$ possible choices, and so on. The $s$ th subgroup is composed of those who have knowledge regarding the inadequacy of $s$ number of alternatives where $0<\mathrm{s} \leqq \mathrm{f}-1$. Under such a simple probability model, Horst developed the formula

$$
P_{i}^{\star}=\frac{1}{T_{i}}\left(R_{i}-W_{i}^{\star}\right)
$$

where $W_{i}^{\star}$ is the number of persons selecting the most frequent incorrect answer. This formula takes into account that there operates partial information under a multiple choice situation and as such is certainly an improvement over the one earlier referred to. However, as Davis [15] points out, although this formula allows for partial knowledge it does not make allowance for wrong answers which are based on misinformation. Davis suggests that when a correction formula is used, it leads to overcorrection if an examinee has misconception, undercorrection if he has partial information and that these two influences tend to cancel out. Solomon [51] has suggested that more models like the one by Horst should be developed which can tackle the problem more adequately by incorporating in the model both partial information as well as partial misinformation. Brownless and Keats [11] have developed a method of estimating the average probability of guessing in an item of a multiple choice type which necessitates a readministration of the test. It might be remarked in this connection that in the case of multiple choice tests, subjects of even the lowest ability have a positive probability, say $1 / k$ of giving a correct answer [where $k \leqq f$ and denotes the average number of effective alternatives] due to the possibility of guessing. Therefore, a plausible form for $P_{i}$ must be one which increases from $1 / k$ to 1 as the ability, $Y$, increases from $-\infty$ to $\infty$.

Another problem associated with the estimation of item parameters is encountered when items are assigned weights of multiple categories before item analysis though such a situation is not common. Most of the item analysis procedures have been developed for items which are dichotomously scored. Gulliksen's [27] entire item selection theory deals with items administered under no time limit condition and scored as either correct or incorrect. Michael and Perry [44] have formulated a theory of item analysis when items are assigned weights of three categories. Mukherjee [46] has brought into attention the difficulties involved in evaluating different items with regard to various item indices in a situation where the responses to an item are scored at multiple level of appropriateness and the maximum possible score differs from item to item in the same test. Since scoring item responses at multiple levels of appropriateness becomes necessary at times, it would be worth while to formulate a generalized theory of item analysis which should also take into account unrestricted scoring of item responses.

The preceding section has dealt entirely with problems involved in the calculation of item difficulty and other item statistics without any special mention of the underlying probability structure. In test theory, a number of probability models have been postulated. These probability models, namely (a) linear model, (b) linear distance model, (c) normal ogive model, (d) logistic function model and (e) arc sine response model, represent the 
basis of conceptualizing the measurement problems ordinarily encountered in the assessment of attitudes, abilities and personality traits. These models are probabilistic in the sense that given the best estimates of the parameters associated with the subject and the item, we can determine the probability with which he will respond in a given way. Inversely, if a best estimate of this probability is available, we can also estimate the parameters pertaining to the subject and item. Torgerson [56] has discussed the first three of these probability models and the estimation procedures involved with each. Basically these models differ with respect to the notion about the trace line for characterizing the items in terms of the underlying attribute the items purport to measure. While the linear model assumes that the ICC can be represented by a straight line, in the normal ogive model, for instance, the trace line of the item is related to the underlying attribute by the integral of the normal probability curve:

$$
f_{i}(x)=\frac{1}{\sqrt{2 \pi}} \int_{-\infty}^{\frac{\left(x-M_{i}\right)}{\sigma_{i}}} \exp \left(-1 / 2 z^{2}\right) d z
$$

where $z=\frac{x-M_{i}}{\sigma_{i}}$.

The mental test theory of Lawley [33] is based upon the normal-ogive model and the assumption of normal ogive trace line can be traced in works as far back as 1936 [Lord (39)]. Besides the notion of normal ogive traceline, the two other important assumptions that are usually made under the normal ogive model are (i) that the unknown (latent) variable $Y$ can be indirectly measured and is normally distributed in the population with zero mean and unit variance and (ii) each item is related to the underlying variable $Y$ but the items are mutually independent of one another when the underlying variable is held constant or partialed out. Under this condition technically known as condition of local independence, it is obvious that the inter-item correlation matrix will have unit rank and hence the model is referred to as Spearman single factor model.

It can be readily seen that when the trace line of item $i$ under the normal ogive model is plotted with the unit expressed in terms of $z_{\text {to }}$, the unit normal deviate corresponding to $P_{i o}$, the trace line becomes a straight line and the equation for the line will be

$$
\text { where } \begin{aligned}
& z_{i g}=a_{i}+b_{i} S_{g} \\
& b_{i}=1 / \sigma_{i} \text { and } a_{i}=-M_{i} b_{i} .
\end{aligned}
$$

The parameter $S_{g}$ refers to the scale value of the $g$-th subject on the latent attribute continuum. The parameter $M_{i}$ is the point on latent attribute continuum where the probability of having positive response is one half and it serves as a location parameter for $i$-th item. The reciprocal of the parameter $\sigma_{i}$ denoted as $b_{i}$ is regarded as the precision or discrimination power of the item $i$.

In the actual estimation of the item parameters $M_{i}$ and $\sigma_{i}$, from the observed proportion of correct item response, neither $P_{i 0}$ nor satisfactory estimates of it are generally available since most of the items are of dichotomous nature and the subject responds to them just once. This difficulty is generally alleviated by dividing the total sample into $g$ subsamples based on the total test score, the assumption being that the expected total 
score is a monotonic function of the underlying attribute. The proportion of people in the $g$-th subgroup who respond positively to $i$-th item is regarded as the best possible estimate of the population parameter $P_{i g} . S_{g}$ therefore denotes the position of the $g$-th subgroup on the latent continuum. It is thus implicitly assumed that within any subgroup, the subjects have the same amount of the attribute under measurement. Given this assumption and the $m n$ observed proportions $P_{i \theta}$ converted into unit normal deviates $z_{i g}$, the problem of estimating item parameters $M_{i}$ and $\sigma_{i}$ for $n$ items can be approached in various ways.

Since Lawley's [33] initial application of maximum likelihood method for estimating the item parameters, several equations have been suggested. The maximum likelihood solution offered by Lord [39] which though constitute the formal solution to the normal ogive model, does not appear very practical. Finney [19] has given a workable solution to this problem. The table prepared by him and Stevens [20] though provide the weighting coefficients and the working value $y_{i}$ for selected values of $a_{i}+b_{i} S_{g}$ the lack of sufficient detail usually necessitates interpolating in tables of normal integral to obtain the values of $P$ and $z$. The weighting coefficient and the values of $y$ must be computed at each point of the ability level within a given iteration. Unless the initial estimates of $a$ and $b$ are very close to the population parameter $\alpha$ and $\beta$, the lengthy computation makes the application of maximum likelihood procedure economically impractical.

Tucker [59] has given an approximate least square solution to the same problem which also requires iterative procedures but it is relatively less time consuming. Tucker [58] has also suggested a simple procedure of estimating the item parameters under the Spearman single factor model without assuming the discriminating power of all the items of the rest equal. He first showed how to solve for the item parameters in terms of the means and variance of a single group from the factorization of inter-itcm terachoric correlation matrix. While it is possible to solve for the item parameters for two subgroups at a time, the basic procedure has not been extended as yet for working with $m$ subgroups where $m>2$.

It may be remarked here that parameters of mental test item typically have been obtained on the assumption that the latent ability obeys a normal ogive distribution. In case of aptitude and achievement tests, empirical results show that the assumption of normal ogive trace line gives a good fit for free response items; a slightly less simple trace line should provide a good fit for multiple choice items. In the special case, where the trace lines are normal ogives and the ability is normally distributed in the group tested, Lord [38, 39] has shown that the ability scale or latent continuum is the same as the common factor of the inter-item tetrachoric correlations.

However, a number of mathematical difficulties arise if the ICC is represented by a normal ogive function. Birnbaum $[4,5,6]$ has shown that these problems can be circumvented if we represent the ICC by a logistic function. He has demonstrated some advantages of adopting the logistic function over the usual normal ogive for ascertaining the probability that an examinee of a given ability would have a speicfied response pattern to a $k$-item test. Baker [2] recently found that in terms of computer running time, the logistic model has a distinct advantage over the normal ogive model. Maxwell [43] has also 
suggested the use of logistic function particularly when maximum likeligood solution of the item parameters is desired. Maxwell [43] has given an iterative method of finding the maximum likelihood estimates of the item parameters. A quick graphical method for estimating these parameters has been provided by Hodges [28].

Some workers have recommended to substitute arc sine distribution in place of the normal distribution. The postulate now goes by the name of arc sine model or the angular response law [Jones and Bock, (31)] in which case instead of transforming the observed proportions into normal deviates, Fisher's arc sine transformation is used. For purpose of variance tests, the arc sine has been found to be very convenient since with angular transformation, the sampling variance stabilizes to a great extent even with small sample size. A maximum likelihood solution assuming angular response law can be obtained in Fisher and Yates [21].

The extension of Lawley's work employing logistic function model appears very promising in shaping the future test theory. One of the important problems of test theory today is the relation between the ability of an individual and his observed test score. Assuming a logistic function, Birnbaum [5] has proposed a workable solution to the problem of estimating the 'true' ability of the individual. While all the different models discussed above have some good properties, real extensive studies have not been taken as yet to test their goodness of fit in estimating the probability of responding positively to an item given the best estimates of the parameters associated with the items as well as individuals tested. Minor researches like making a comparative study of the power properties of the asymptotic tests using normal approximation and $\sin -1 \sqrt{p}$ transformation for testing the equality of two observed proportions have not been taken as yet.

Item selection procedures under different probability models:

A basic problem in test construction is the suitable selection of $k$ out of $N$ test items, scores on the $k$ items to be used in predicting the unknown ability or latent trait $Y$, for the individuals taking the test. Customarily, items having high correlation with the total score or with a suitable external criterion are selected. Under the situation where the matrix of inter-item corrlation matrix is of unit rank (Spearman single factor model) and the items are of equal difficulty level, items having high inter-item correlation can justifrably be selected, since it can be shown explicitly that the test validity $\varrho_{x y}$ which here is the correlation coefficient between the test score and common factor of the test items, increases with increasing inter-item correlation $\varrho_{i j}$ when all the items are symmetric, i.e. when all the items are equally difficult and the inter-item correlations are the same. This monotonic increase in $\varrho_{x y}$, however, stops at a point and then it decreases to the value $\sqrt{2 / \pi}$ $=.798$ [Sitgreaves (48)]. Thus Tucker [57] has shown that theoretically it is impossible to have a very high validity for a test composed of equivalent items whose reliability is perfect. Loevinger [36] has named this phenomenon in mental test design as "the attenuation paradox" though" though this was observed by several other workers notably by Gulliksen, Tucker [57] and Brogden [9] long before her.

One possible reason of the so called 'attenuation paradox' as pointed out by Lord [40], and more recently by Solomon [51] and Sitgreaves [48] is the inappropriate use of the cor- 
relation coefficient as a measure of validity since the regression of test score $x$ on $Y$, is certainly curvilinear [Brogden (9), Lord (38)] whose curvature increases with increasing $\varrho^{\star}$, where $\varrho^{\star}=\varrho_{i j}{ }^{\star}$ and $\mathrm{i}, \mathrm{j}=1,2, \ldots n^{1}$. The curvature !becomes maximum where the validity $\varrho_{x y}$, reaches its maximum. Lord [40] and Solomon [51], therefore, suggested the use of correlation ratio $\eta$, which would satisfy the curvilinear regression in order to overcome the paradox. Using the curvilinear correlation coefficient $\eta$, as a measure of test validity, Solomon [53] attempted to study if the paradox could be eliminated for equivalent items. He found that while the use of $\eta$, "moved the maximum validity to correspond to a higher value of, it did not remove the paradox" [Solomon, (53, p.336)]. It is interesting to note that though Tucker [57], Lord [40], Birnbaum [5] and more recently Solomon [51] have explicitly expressed the opinion that the so called paradox will not appear with a test composed of nonequivalent items, none of them has given any mathematical proof of the same. Empirical studies on this line are also clearly lacking. It should be also determined whether the paradox appears where the assumption of linear multifactor model for the equivalent items holds. It appears intuitively that the paradox cannot be avoided even employing linear multiple factor model or any other probability model for the item responses. This has to be confirmed mathematically.

Basic to the question of item selection is the problem of obtaining a suitable criterion in terms of which the discriminating power can be judged. In the field of personality measurement, for example, no suitable criterion measure exists for traits like dominance, introversion, emotional stability etc. and often item selection for assessing such traits is based on the criterion of internal consistency. Items having high correlations with the total score are selected which often results in high inter-item correlation. This condition is not conducive to efficient prediction in the field of personnel selection. In so far as occupational success is dependent on the possession of certain specific and unrelated traits, this method tends to reject just those items in which we are most interested. Loevinger, Gleser and DuBois [37] have developed a procedure of maximizing the discriminating power of a multiple score test. The procedure involves maximizing the homogeneity of each subtest and minimizing the intercorrelation between the items of a subtest. It gives a formal solution to two problems: when to stop adding items to a cluster and when two separate clusters should be combined. The method also provides an answer to the question raised by Tukey [60] as to what should be done when the items are large and correlated. However, the problem of item selection for validity which Tukey [60] included among the unsolved problems of experimental statistics is not very adequately solved mainly because of the difficulty in obtaining suitable criterion. The questions relating to the validity of the test as well as validity of individual items are very important but extremely difficult to resolve. "Not only is a measure of association between two sets of variables required, but a complicating condition arises, since the set of test variables is observable (manifest) and the other set is composed of unobservable (latent) variables" [Solomon, (53, p.331)].

However, if a suitable external criterion becomes available and we wish to select items

1. $\varrho_{i j}$ indicates the inter-item correlations from the observed test scores whereas $\varrho_{i j}{ }^{\star}$ denotes the interitem correlations determined from the underlying probability model. 
which can measure different dimensions of the criterion, the Spearman single factor model has to be replaced by the linear multifactor model. This model is much more realistic than the former and at the same time can be extended to treat the Spearman single factor model as a special case. Under the situation where the linear multifactor model holds, Elfving, Sitgreaves and Solomon [18] have shown that the usual item selection process based on maximum criterion correlation $\left(\varrho_{x y}\right)$, may be quite poor. However, if the Spearman single factor model underlies the item responses, then the two criteria of item selection, i.e., highest item-criterion correlation and minimum mean squared error of estimate, minimum $(\hat{z}-z)^{2}$, will lead to the same item selection result. In Elfving's [17] linear multiple factor model, besides the $n$ manifest set of variables $X_{1}, X_{2}, \ldots, X_{n}$, there is a criterion variable $z$ to be estimated from the $x$ 's that is also assumed to a linear function in the component of $Y$ and quite natuarally assumed to be composed of the same number of components of $Y$ as the $X$ 's. Thus the given set of possible observations, i.e., the item responses are of the form

$$
X=a_{i 1} Y_{1}+a_{i 2} Y_{2}+\ldots+a_{i k} Y_{k}+\theta_{i},(i=1,2, \ldots, n ; k<m<n)
$$

and the criterion score of individual $g$,

$$
z_{g}=c_{1} Y_{1 g}+c_{2} Y_{2 g}+\ldots+c_{k} Y_{k g}+e_{g},(g=1,2 \ldots, N)
$$

where the coefficient vector $a$ and $c$ are known and the parameter vector $Y$ is unknown and the error $\operatorname{term} \theta$ are uncorrelated random variables with mean zero and unit variance. The term $e$ is also uncorrelated with the $\theta \mathrm{s}$ and has variance $\sigma_{e}^{2}$.

Under this model, Elfving et al. [18] have shown how it is possible to select the best $m$ out of $n$ predictors of a criterion without computing ( $n k$ ) multiple correlations by restricting $z$ to unbiased linear prediction in the $X$ 's,

$$
z_{o}=\sum_{i=1}^{m} q_{i} x_{i \sigma}
$$

where $m$ is the number of items in a set under examination and the set has been selected from a large pool of $n$ itens. The solution of $z$ satisfies both $E(\hat{z}-z)=0$ and $E(\hat{z}-z)^{2}$ $=$ minimum.

One obstacle in the use of the multifactor model for actual item selection is that the factor loadings for the items must be known beforehand. While it is possible to estimate them by making a realistic guess about them in many situations from data collected by an administration of the items, a larger obstacle presents itself because the factor loadings for the criterion variable $z$, namely the coordinates of the row vector are unknown and very difficult to estimate.

Thus, we are faced with real practical problems if we employ either the Spearman single factor model or replace it by the linear multiple factor model. It seems quite reasonable therefore to explore whether we can achieve anything new in the methodology of test design if no assumption or very few assumptions are made about the underlying trait and item responses and we consider only an item variable and a criterion variable both of which are dichotomous in nature. Raiffa [47] has explored this non-parametric situation in more detail within the framework of statistical decision theory. Solomon [52] has considered item selection procedure in an actual illustration involving dichotomous items, Applying the method developed by Bahadur [1], he has made use of the likeliood ratio pro- 
cedure for the purpose of classifying individuals into one of the two groups. Here, even for very small $k$, say $k=4$, and a pool of ten items, the matter of choosing the best form out of ten items is a very tedious process. However, the whole field of item selection under various non-parametric situations seems relatively unexploited and much can be done in this area with the access of a high speed electronic computer.

One interesting problem relating to item selection procedure that suggests itself under a non-parametric situation is that of finding an optimum point in the criterion score continuum at which the item will show maximum discrimination and yet the instability of the resulting value due to the exclusion of the cases in the middle of the distribution will be minimum. It is nearly customary in item analysis to follow Kelley's [32] method of analysis based on 27 per cent upper and lower groups only dropping out 46 per cent cases falling in the middle. While Kelley [32] has given mathematical justification of the underlying rule and not long ago Cureton [13] presented a simplified derivation of the formula, it is seen that a $27 \%$ truncation at both sides will lead to the minimum standard error of the correlation only when the correlation is zero in the poulation. Thus the popular 27 per cent truncation has no meaning whatsoever. Even if it has some utility, the rule holds only where the normality assumption regarding the criterion score distribution is justified. It is therefore, necessary to determine what would be the rule where the criterion score obeys different types of distribution other than normal.

\section{Problems Associated with the Measures of Worth in Test Design}

In comparing two psychological tests for purpose of evaluation or selection, it is necessary to choose certain operating characteristics of the tests as a measure of worth in terms of which comparison can be made. Traditionally reliability $\left(\varrho_{x y}\right)$ and validity $\left(\varrho_{x y}\right)$ of a test in the form of correlation coefficient have been the indices of worth for the test. While the correlation coefficient has some pragmatic value for tests of mental ability, Lord [40], Solomon $[51,53,54]$ and Sitgreaves [48] have shown the inadequacy of representing the measure of relationship between ability $y$ and total test score $x$, in the form of a correlation coefficient. Reliability and validity coefficients representing an overall index of discrimination do not apply at all points along the score scale. Levine and Lord [35], therefore, have suggested the use of $D x$ as the index of discriminating power of a test at score $x$ which is given by the ratio

$$
D x=\frac{b y / x}{S y \cdot x}
$$

where $x$ is measured in sigma units, $b y / x$ is the slope at raw score $x$ and $S y . x$ is the s.d. of ability, $y$, at a given level of test score $x$.

Berkson [3] has also criticized the traditional use of biserial or tetrachoric correlation in evaluating the effectiveness of a test for classifying individuals to either of dichotomous groups. He has developed a method of determining the utility, defincd as the fraction of individuals actually belonging to category $A$ which the test correctly designated as $A$ and the cost defined as the fraction of individuals actually belonging to category $B$ which the test incorrectly designates as $A$. 
Sitgreaves [48] has shown that even under a situation where the test items are symmetric, a situation where the Spearman single factor model operates between the items and underlying trait, reliability and validity coefficients are not necessarily the best measures of the goodness of a test, since their use results in the so-called "attenuation paradox".

Lord [40] also has commented on the inadequacy of an overall coefficient of validity. As he writes,

"It is too much to ask that the different discriminating powers of a single test for a wide variety of examinees and with respect to various unspecified decision problems should all be harmoniously and nonparadoxically summarized in a single 'validity' coefficient. ..... Such a composite coefficient is not basic to psychometrics. The discriminating power of the test for a specified decision problem..... is the truly basic concept..... If the problem is properly formulated, all of modern theory of decision-making and theory of estimation and testing hypotheses can be brought to bear" [Lord, (40, p.507 and 509)].

Clearly we should take into account the question that the test results are to aid in answering while selecting an adequate measure of worth to evaluate the usefulness of a test. If the purpose of the test is to select a number of individuals from a population $Y=y$ values greater than the ability level $\alpha$ which may be true in civil service examination or for admission in a professional school, the use of a test composed of symmetric items may be quite justified even if the validity of the test is low but has a high reliability. On the other hand, this type of test is not particularly helpful if it is desired, for instance, to rank a number of individuals from a population according to their position on the $Y$ continuum.

If the basic decision to be made about individuals from a population is clearly specified such that we may be able to quantify losses associated with diverse wrong decisions when $Y=\gamma$, then the minimum expected loss to be achieved with the use of a given test is a natural index for measuring the utility of the test.

In many situations, however, it is not possible to specify the decision so sufficiently and only a particular decizion is specified when $Y=y$ such as deciding whether or not a student should be admitted to the school. In such situations it seems reasonable to use the item responses to estimate the individual $Y$-value; then assume this is the exact value of $Y$ and make the desired decision. Based on this resoning, Sitgreaves [49] has adopted and examined in detail the use of an index based on minimum error variance criterion, i.e., the index that minimizes the mean squared error of estimate and indicates the degree of precision or closeness with which the estmation of $Y$-value has been made. Thus if $y=f(x, x, \ldots x)$ is the estimate of the value of $Y$ when $Y=y$, then the expected squared error of estimates is $\mathrm{E}(\hat{y}-\gamma)^{2}$. If we estimate $Y$ by its population mean value of zero, without taking into consideration any test results, the expected squared error of estimate is simply the population variance which is 1 . Thus, any admissible procedure of estimation must have an expected squared error that does not exceed unity, since it must do better than when the mean value of $Y$ for $y$ is used without taking into account any test results.

Sitgreaves [49] examines this measure of worth in a restricted situation where the observable dichotomous variables, i.e., the item responses, all measure the same underlying variable $x$, a criterion to be satisfied for constructing Guttman scale. She therefore, assumes that the distribution of test score is equivaltnt to the joint discribution of iteni responses. 
In this case, a $k$-item test is defined by $k+1$ numbers, i.e., the correlation between $X$ and $Y$ which is denoted by $\sqrt{\varrho^{\star}}$ and the numbers $\alpha_{1}, \alpha_{2}, \ldots \alpha_{k}$ related to the item difficulties. For a given test, the estimating procedure for $Y$ that minimizes the expected squared error of estimate is then determined and the value of this minimum, say $\tilde{S}^{2}\left(\varrho^{\star}, \alpha_{1}, \alpha_{2}, \ldots \alpha_{k}\right)$ is found. Actually as a measure of worth of the test, Sitgreaves uses 1 minus this minimum value, i.e.,

$$
h\left(\varrho^{\star}, \alpha_{1} ; \alpha_{2}, \ldots \alpha_{k}\right)=1-S\left(\varrho^{\star}, \alpha_{1}, \alpha_{2}, \ldots \alpha_{k}\right) .
$$

This number ranges from 0 to 1 and a higher value of $h$ indicates more efficiency of a test. The index provides us with a means of comparing tests of different lengths, as well as tests of the same length but with different values of the parameters $\varrho^{\star}{ }_{1 y}, \varrho^{\star}{ }_{2 y}, \ldots, \varrho^{\star}{ }_{k y}$; $\alpha_{1}, \alpha_{2}, \ldots, \alpha_{k}$. Sitgreaves [49] has shown that the index $h$ can be considered as the population value of the square of a correlation ratio $\eta^{2}$ between the latent variable and manifest set of variables. She has shown that within this restricted but classical multivariate model, the index $h$ is the product of two meaningful factors. The first factor depends only on the observables and is a measure of reliability. The second factor is a correlation (validity coefficient) between the continuous variable $Y$ and the manifest variable $X$. Thus both reliability and validity must be higher in order for the goodness of the test, $h$ to be high. In addition the use of this index enables the psychometrician to avoid the attenuation paradox faced by previous workers. It also becomes possible in the restricted situation studied by Sitgreaves to select directly the characteristics of the optimal item. For example, given that a test should contain $k$ number of items, the item difficulty can be obtained explicitly. The inter-item correlations are already determined by the very restricted nature of the model.

Sitgreaves [50] has examined the same index, $h$, under a less restricted situation too in which case she does not assume that the test items satisfy a Guttman scale i.e., all the test items are tapping the same underlying attribute. Instead she specifically assumes that each item is measuring a different latent trait from $X$. For the case of two-item test, she demonstrates that the index $h$, is uniformly higher for any test within the general classical model than for any two-item test in this model which in addition satisfies a Guttman scale. We can expect the same results with a $k$-item test but since tables to obtain the integrals over the $k$-variate normal distribution are required for the purpose and tables now exist for $k=3$, it is not possible to demonstrate the merit of this measure for a test even of four items. In predicting the criterion from the pattern of the examinee's responses rather than from a single test score, Sitgreaves [50] finds it unwieldy to work with longer tests since as the number of items increases, the number of possible response patterns increases by leaps and bounds. With a test of 25 items, for example, there are more than 33 million possible response patterns. One way out of this is to assume a logistic item characteristic curve instead of a normal ogive one. With this change, Birnbaum [4] has shown that the response pattern contains no information beyond that contained in a single appropriately defined test score.

2. The notation $\sqrt{\varrho^{\star}}$ is used here since in general case $Q^{\star}$ is employed to define inter-item correlation between equivalent items. The square root of $\varrho^{\star}$, in the general case would then define item validity, the item's correlation with the latent variable $Y$. 
While Sitgreaves $[49,50]$ uses $h$ as an index for measuring the goodness or worth of a test which has been used by Elfving et al. [17] too but for a different underlying probability structure, Birnbaum [4] suggests that the length of the confidence interval, $U(t, \alpha / 2)-$ $s(t, \alpha / 2)$ for an examinee's ability $y$ based either on his $s$, the unweighted sum of item scores or $t$, the weighted sum of item scores, be used as a measure of the amount of information provided by the test $X$. Birnbaum [5] has shown that "if the $\varrho^{\star}$ values are nearly equal then $s$ must be virtually as efficient as $t$, but if $e^{\star}$ values vary greatly within a test, then $s$ must be much less efficient than $t$ " (Birnbaum, (5, p.3)]. Lord [39] earlier demonstrated that the usual type of test score, i.e., the total number of correct responses, is a sufficient statistics [Mood, (45, p.151)] for estimating the examinee's ability in the case of a test composed of equivalent items and thus is the most adequate summarization of the item responses. However, when a logistic distribution is assumed for the latent continuum, $Y$, Birnbaum [4] showed that a more general yet similar statement like the one made by Lord can be made. Under a logistic function model even when the items are not equivalent but all of dichotomous nature, a linear function of the item responses, in which the weights depend on item validity, is a sufficient statistics. For then

$$
t=w \sum_{i=1}^{k} \frac{\varrho_{i j}^{\star} S_{i}}{\sqrt{1-\varrho_{i j}^{\star 2}}}
$$

where the item responses, $S$ receive a score of one or zero. Thus under this situation, $t$ becomes a sufficient statistics ( $w$ is a constant). It may be noted here that the appropriate weights depend upon the item validities. If the items are equivalent, then $t$ is the test score multiplied by a constant, the same result as in the normal distribution situation for equivalent items.

It is clear then that the simple sum or simple weighted sum of item responses has a desirable property in some restricted situations where heavy assumptions are made about the distribution of the underlying trait, $Y$. Thus the traditional notion of test score namely the number of correct responses "can be given a quite comfortable place within the logistic test model, although this will not occur generally with other test models [Birnbaum, (4, p.15)]. Similarly an overall index of test validity in the form of correlation coefficient is a meaningful measure of worth only when the Spearman single factor assumption holds true. It is therefore, high time that the product moment correlation which is employed to define both reliability and validity of a test be replaced by some other measures of worth like the ones proposed by Sitgreaves or by Birnbaum which are more meaningful and less paradoxical.

\section{Problems in the Modern Decision Theory Framework}

Like personnel selection or classification, the item selection procedure may be also viewed as a decision process. We can also evaluate the risk, benefit, or utility of various courses of action taken in selecting $n$ sets of items from a pool of $N$ sets. Raiffa [47] has already made an excellent start in this regard and for the sake of simplicity has chosen a test variable and a criterion variable both of which are dichotomous. The unknown criterion variable is considered as a classificatory variable whose value specifies the two 
possible classes to which an individual can belong. The two values of the classificatory variable is treated to represent two possible states of nature while the two possible classificantions of the individual represent two possible terminal actions. The problem of item szlec ion has been conceived of as analogous to making comparison of several experiments each parf rrmed when a set of items are chosen. In the light of modern decision theory, Raiffa considers the problem of item selection under two heads: first when the total number of available irem; is sm?ll such as in medical diagnosis and second when it is de ired to select a snall number of items from a large pool of potential items.

With regard to the second type of situation, Raiffa, however, has shown that when the potential number of item pool is large, say 100 , and it is desired to choose even 25 out of these, serious conceptual problem arises and the computational task becomes overwhel.mingly difficult. The conceptual difficulty arises mainly with large number of items as it becomes increasingly difficult to assume that we have enough initial experience with a population of individuals to assign meaningful joint probabilities to the $2^{25}$ possible response patterns. In practice, we usually have initial experience with a group of individuals who are given $m$ items and from the initial data we can estimate the joint distribution of any subiet of item; however, the relizbility of any estimate rapidly deteriorates as the number of item; iacreases.

Be:ide these problems, we have a number of other problems connected with the evaluation of benefit obtained from tests some of which have been touched in our discussion of the masures of worth. Cronbach and Gleser [14] have also discussed some problems in this connection such as the relation of benefit (utility) to validity coefficient, evaluation of a test in the light of its independent contribution to utility over and beyond which is available from the other easier sources, the benefit of tesing under fixed and adaptive treatments, the marit of taking sequential strategies by repeated testing etc. The same problems can be taken with regard to the evaluation of each individual items if computational facilities are available and thereby add something new in the item selection procedure. We can obtain information curves for individual items like the ones shown by Birnbaum [6] and study the loss in the efficiency of a test item for measuring different types of underlying trait or loss in the efficiency of a test when optimal weighting of item responses is ignored. We can also obtain risk curves for a set of selected items to indicate the probability of misclassifying individuals into one of the dichotomous criterion groups given a cut-sff point with respect to a particular criterion. Birnbaim and Maxwell [7] have suggested a method of obtaining the conditional probability of misclassication where classification is based without assuming anything about possible forms of distribution and the problem is to classify individuals into $k$-groups where $k>2$ and test results on $m$ variates are available for each individual. Solomon [52] also has recently explored the worth of several classification procedures based on item response vectors as well as total scores (unweighted sum of the item responses) for different sets of items of an attitude questionnaire in a situation where the available criterion (I.Q.scores) was considered as dichotomous and where for each response vector, the number of individuals giving the response is known for each of the two classes. Solomon's [52] risk curves showing the probability of misclassification of type $A$ against the probability of misclassification of type $B$ for different 
sets of items make it possible to make valuable decisions in test design-namely, how many items and what type should be used in a particular study.

\section{Summary AND Conclusions}

A number of problems in the design of psychological tests arising from the various assumptions regarding the probability structure of the item responses and the underlying trait have been discussed. It is concluded that in designing a test for a given decision problem, special considerâtion should be given (i) to determine what could be the best procedure of using test responses which are statistically efficient for the given application, (ii) to develop a suitable measure of worth for the test as well as for the test items and study their properties, and (iii) to base item selection procedure in the light of the above measure of worth the efficiency of which has been shown to be better than other measures under the given situation.

In this connection, some of the important problems which merit attention are the following:

(a) the statistical estimation of difficulty values and other parameters of multiple choice items under different probability models connected with the distribution of the guessing variable, (b) a generalized theory of item analysis taking into account unrestricted item scoring method, (c) statistical estimation of 'true' ability of the examinee assuming different types of probability structures governing the ability in question and making a test of goodness of fit for each, (d), development of some more mathematical models for finding and evaluating optimum test design and lastly (e) item selection procedure under non-parametric situations. The whole field of item selection should be reconceptualized from an utility standpoint and as such the machinaries of decision theory should find more and more proper exploitation in the technique of designing optimum tests.

\section{REFERENCES}

1. Bahadur, R.R., A representation of the joint distribution of response to $n$ dichtomous items, in Studies in Item-analysis and Prediction, H. Solomon (Ed), Stanford: Stanford Univ. Press, 1961, Chapt. 9.

2. Baker, F.B., Empirical comparison of item prarameters based on the logistic and normal functions, Psychometrika, 1961, 26, 239-246.

3. Berkson, J., "Cost utility" as a measure of the efficiency of a test, J. Amer. Statist. Assocn., 1947, 42, 246-255.

4. Birnbaum, A., Efficient design and use of tests of mental ability for various decision making problems, USAF Series in Statistics, Report No. 58-16, Randolph Air Force Base, Texas: School of Aviation Medicine, January 1957.

5. Birnbaum, A., On the estimation of mental ability, Series Report No. 15, AFB

Randolph Field, Texas: School of Aviation Medicine, 1957.

6. Birnbaum, A., Further considerations in tests of a mental ability, USAF Technical Report No. 17, AFB Randolph Field, Texas: School of Aviation Medicine, 1957.
7. Birnbaum, A., and Maxwell, A.E., Classification procedures based on Bayes' formula, Technical Report No. 4, Brook AFB, Texas: School of Aviation Medicine, July 1959.

8. Bock, R.D., Components of variance analysis as a structural and discriminal analysis for psychological tests, Brit. J. Stat. Psychol., 1960, 13, 151-63.

9. Brogden, H., Variation in the test validity within variation in the distribution of item difficulties, number of items and degree of their intercorrelations, Psychometrika, 1946, 11, 197-214.

10. Brogden, H.E., An approach to the problem of differential prediction, Psychometrika, 1946, 11, 139-154.

11. Brownless, V.T., and Keats, J.A., A retest method of studying partial knowledge and other factors influencing item response, Psychometrika, 1958, 23, 67-73.

12. Carter, H.D., How reliable are the common measures of difficulty and validity of objective test items, J. Psychol, 1942, 13, 31-39.

13. Cureton, E.E., The upper and lower twentyseven per cent rule, Psychometrika, 1957, 
22, 293-296.

14. Cronbach, L.J., and Gleser, G.C., Psychological tests and personnel decisions, Urbana, Ill., Univ. of Illinois Press, 1957.

15. Davis, F.B., Item analysis in relation to educational and psychological testing, Psychol. Bull., 1952, 49, 97-121.

16. Dunn, T.F., and Goldstein, L.G., Test difficulty, validity and reliability as functions of selected multiple choice item construction principles, Educ. Psychol. Measmt., 1959, 19, 171-179.

17. Elfving, G., Item selection and choice of nonrepeatable observations for estimation, Proceedings of the Third Berkeley Symposium on Mathematical Statistics and Probability, J. Neyman, (Ed), Berkley, Calif.: Univ. of California Press, Vol. 1, 1956.

18. Elfving, G., Sitgreaves, R., and Solomon, $H$., Item selection procedures for item variables with a known factor structure, Psychometrika, 1959, 24, 189-205.

19. Finney, D.J., The application of probit analysis to the results of mental tests, Psychometrika, 1944, 9, 31-39.

20. Finney, D.J., and Stevens, W.L., A table for calculating of working probits and weights in probit analysis, Biometrika, 1948, 35, 191-201.

21. Fisher, R.A., and Yates, F., Statistical tables for biological, agricultural and medical research, 4th ed., New York: Hafner, 1953.

22. French, J.W., A technique for ctiterion-keying and selecting test items, Psychometrika, 1952, 17, 101-106.

23. Gadel, M.S., The relationship of item validity shrinkage to curvilinearity of response distributions, Educ. Psychol. Measmt, 1958, 18, 145-152.

24. Gibbons, C.C., The predictive value of the most valid items of an examination, J. educ. Psychol., 1940, 31, 616-621.

25. Guilford, J.P., Psychometric Methods, New York: McGraw-Hill, 1954.

26. Guilford, J.P., Factor analysis in a test development program, Psychol. Rev., 1948, 55, 79-91.

27. Gulliksen, H., Theory of mental tests, New York: Wiley, 1950.

28. Hodges, J.L., Jr., Fitting the logistic by maximum likelihood, Biometrics, 1958, 14, 453-461.
29. Horst, P., The difficulty of a multiple choice test item, J. educ. Psychol., 1933, 24, 229232.

30. Horst, P., Optimal test length for maximum differential prediction battery, $P_{s y-}$ chometrika, 1956, 21, 51-67.

31. Jones, L.V., and Bock, R.D., Methodology of preference measurement, Report No. 4, Contract DA 19-129, Quartermaster Food and Container Institute for the Armed Forces, Chicago, Ill., September 1957.

32. Kelley, T.L., The selection of upper and lower groups for the validation of test items, J. educ. Psychol., 1973, 30, 17-24.

33. Lawley, D.N., On problems connected with item selection and test construction, Proceed. Royal Soc., Edinburgh, 1943, 61-A, Part 3, 273-287.

34. Lawshe, C.H., and Harris, D.H., The method of reciprocal averages in weighting personnel data, Educ. Psychol, Measmt., 1958, 18, 331-336.

35. Levine, R., And Lord, F.M., An index of the discriminating power of a test at different parts of the score range, Educ. Psychol. Measmt., 1959, 19, 497-503.

36. Loevinger, J., The attenuation paradox in test theory, Psychol. Bull., 1954, 51, 493-504.

37. Loevinger, J., Gleser, G.C., and DuBois, P.H., Maximizing the discriminating power of a multiple score test, Psychometrika, 1953, 18, 309-317.

38. Lord, F.M., A Theory of Test Scores, Psychometric Monog., 1952, No. 7.

39. Lord, F.M., An application of confidence intervals and of maximum likelihood to the estimation of examinee's ability, Psychometrika, 1953, 18, 57-76.

40. Lord, F.M., Some perspectives on "the attenuation paradox in test theory", Psychol. Bull., 1955, 52, 505-510.

41. Lord, F.M., Problems in mental test theory arising from erros of measurement, J. Amer. Statist. Ass., 1959, 54, 472-479.

42. Lumsden, J., The construction of unidimensional tests, Psychol. Bull., 1961, 58, 122131.

43. Maxwell, A.E., Maximum likelihood estimates of item parameters using the logistic function, Psychometrika, 1959, 24, 221-227. 
44. Michael, W.B., and Perry, N.C., A theory of item analysis based on the scoring of items at three levels of appropriateness, Educ. Psychol. Measmt., 1955, 15, 323-336.

45. Mood, A.M., Introduction to the Theory of Statistics, New York: Wiley, 1950.

46. Mukherjec, B.N., Item analysis when item responses receive differential creidts, J. Vocational Educl. Guidance, 1959, 5, 140-47.

47. Raiffa, H., Statistical decision theory approach to item selection for dichotomous test and criterion variables, Report No. 56-139, AFB Randolph field, Texas: School of Aviation Medicine, January 1957.

48. Sitgreaves, R., A statistical formulation of the attenuation paradox in test theory, Report No. 57-1, AFB Randolph Field, Texas: School of Aviation Medicine, August 1956.

49. Sitgreaves, R., Optimal test design in a special testing situation, Report No. 57-117, AFB RAndolph Field, Texas: School of Aviation Medicine, September 1957.

49. Sitgreaves, R., Optimal test design in a special testing situation, Report No. 57-117, AFB RAndolph Field, Texas: School of Aviation Medicine, September 1957.

50. Sitgreaves, R., Further contributions to the theory of test design, In Studies in item analysis and prediction, H. Solomon, (Ed), Stanford, Calif: Stanford Univ. Press, 1961, Chap. 3.

51. Solomon, H., Iten analysis and classification techniques, In Proceedings of the Third Berkeley Symposium on Mathematical Statistics and Probability, Berkley and Los Angeles: Univ. California Press, 1956.
52. Solomon, H., Classification procedures based on dichotomous response vectors, Contribution to probability and statistics, Stanford, Calif: Stanford Univ. 1960.

53. Solomon, H., Measures of worth in Item analysis and Test design, In Mathematical methods in the social sciences, K.J. Arrow, S. Karlin and P. Suppes (Eds), Stanford, Calif: Stanford University Press, 1960.

54. Solomon, H., (Ed) Studies in item analysis and prediction, Stanford, Calif: StanfordU niv. Press, 1961, Introductory Chapter.

55. Thorndike, R,, Reliability, In Edcuational measurement, E.F. Lundquist (Ed), Washington, D.C., American Council on Education, 1951, Pp. 560-620.

56. Torgerson, W.S., Theory and methods of scaling, New York; Wiley, 1958.

57. Tucker, L.R., Maximum validity of a test with equivalent items, Psychometrika, 1946, 11, 1-13.

58. Tucker, L.R., A method for scaling ability test items in difficulty taking the item unreliability into account, Amer. Psychologist, 1948, 3, 309-310 (Abstract).

59. Tucker, L.R., A level of proficiency scale for a unidimensional skill, Amer. Psychologist, 1952, 7, 408-408 (Abstract)

60. Tukey, J.W., Unsolved problems of experimental statistics, J. Amer. Statist. Ass., 1954, 49, 706-731.

61. Webster, H., Maximizing test validity by item selection, Psychometrika, 1956, 21, 153-64.

62. Webster, H., Item selection method for increasing test homogeneity, Psychometrika, 1957, 22, 395-403.

Bishwa Nath Mukherjee (1933- ) MA 1953 Univ. of Patna. B.M. Institute of Child Development, Ahmedabad 6. 1961-62 Fulbright scholar in the U.S. 